\title{
Cross-modular processing in a spiking neural network model
}

\author{
Diogo PC Vieira*, Antonio C Roque \\ From Twentieth Annual Computational Neuroscience Meeting: CNS*2011 \\ Stockholm, Sweden. 23-28 July 2011
}

According to the classical view about cortical organization, primary sensory areas are exclusively dedicated to a single sensory modality. Recent studies have challenged this view by showing that primary sensory cortices may undertake cross-modal processing whenever necessary $[1,2]$. Despite the growing interest in cross-modal cortical processing, the neural basis of this phenomenon remains largely unknown. How to understand cross-modal cortical processing given the anatomical structure of the cortex and the dynamic features of cortical neurons? It is known that cortical architecture is modular, so that neurons within a module are more densely connected among themselves than with neurons in other modules [3], and that the specificities of cortical connections depend on their range, with excitatory connections reaching longer distances than inhibitory connections [4]. It is also known that cortical neurons belong to different electrophysiological classes [5].

In this work we used a modular network of spiking neurons to study cross-modular processing in terms of average spike rate and latency. The network was made of 1,000 neurons modeled according to the Izhikevich formalism [6] to simulate three electrophysiological classes: fast spiking (FS), regular spiking (RS) and bursting (BS) neurons. Synaptic connections were modeled by $\alpha$-functions. To generate the modular network, we started with a random network with a 4:1 ratio of excitatory to inhibitory neurons and sparse connectivity. The network was randomly divided into four modules of equal size. Each existing inter-modular inhibitory connection was deleted and corresponding intra-modular inhibitory connections were randomly created in turn. A similar rewiring was made for the inter-modular excitatory connections but with

\footnotetext{
* Correspondence: diogopcv@gmail.com

Departamento de Física, FFCLRP, Universidade de São Paulo, Ribeirão Preto, SP, 14040-901, Brazil

Full list of author information is available at the end of the article
}

probability $p$ of deleting an excitatory inter-modular connection. Furthermore, two modules (let us call them modules 1 and 2) were randomly selected and all connections between them were deleted. Three experimental protocols were performed: (a) the entire network was stimulated by random noise for $1200 \mathrm{msec}$; (b) similar to (a) but module 1 also received a fixed input pattern from $t=200 \mathrm{msec}$ to $\mathrm{t}=250 \mathrm{msec}$; and (c) the only input given to the network was the fixed pattern to module 1 only for the same time interval as in (b).

The raster plots of the four modules for protocol (a) show a similar frequency response for all of them but the spiking activity of module 2 cells is out of phase in comparison with activities in other modules. In the case of protocol (b), the presentation of the fixed input pattern to module 1 caused a slight and sustained increase in the average firing frequency of all modules. The start of this increase is delayed by $15 \mathrm{msec}$ for modules 3 and 4 , which are directly connected with module 1 , and by 30 msec for module 2 , which is only indirectly connected with module 1 . These delays are not only due to synaptic delay (equal to $1 \mathrm{msec}$ ) but may also be due to intrinsic network properties. For protocol (c) the situation was similar to protocol (b) but with a lower average spike rate which decays toward zero after offset of the external stimulus. The simple network studied allow propagation of information between modules, with modules that do not receive direct external input responding stably (with delays) to an external and fixed input pattern presented to a single module. This suggests that more detailed analyses of modular networks may help understanding of cross-modal processing.

\section{Acknowledgements}

Work supported by CNPq.

Published: 18 July 2011 


\section{References}

1. Falchier A, Clavagnier S, Barone $\mathrm{P}$, Kennedy $\mathrm{H}$ : Anatomical evidence of multimodal integration in primate striate cortex. J Neurosci 2002, 22(13):5749-5759.

2. Lakatos P, Chen CM, O'Connell MN, Mills A, Schroeder CE: Neuronal oscillations and multisensory interaction in primary auditory cortex. Neuron 2007, 53(2):279-292.

3. Sporns O, Chialvo D, Kaiser M, Hilgetag C: Organization, development and function of complex brain networks. Trends Cogn Sci 2004, 8(9):418-425.

4. Bosking WH, Zhang WH, Schofield B, Fitzpatrick D: Orientation selectivity and the arrangement of horizontal connections in tree shrew striate cortex. J. Neurosci 1997, 17(6):2112-2127.

5. Contreras D: Electrophysiological classes of neocortical neurons. Neural Networks 2004, 17:633-646.

6. Izhikevich EM: Simple model of spiking neurons. IEEE Trans Neural Networks 2003, 14(6):1569-1572.

doi:10.1186/1471-2202-12-S1-P270

Cite this article as: Vieira and Roque: Cross-modular processing in a spiking neural network model. BMC Neuroscience 2011 12(Suppl 1):P270.

\section{Submit your next manuscript to BioMed Central} and take full advantage of:

- Convenient online submission

- Thorough peer review

- No space constraints or color figure charges

- Immediate publication on acceptance

- Inclusion in PubMed, CAS, Scopus and Google Scholar

- Research which is freely available for redistribution

Submit your manuscript at www.biomedcentral.com/submit 